\title{
Traitement Chirurgical Des Eventrations Abdominales Avec Pose De Prothese: Experience A Court Terme Du Chu Yalgado Ouedraogo
}

\author{
Zaré Cyprien \\ Service de Chirurgie Générale et Digestive, \\ CHUSS Bobo-Dioulasso, Burkina Faso \\ Traoré Sékou \\ Ouangré Edgard
}

Service de Chirurgie Générale et Digestive, CHUYO, Burkina Faso

\section{Belemlilga Hermann}

Service de Chirurgie Générale et Digestive, CHUSS Bobo-Dioulasso, Burkina Faso

\section{Zida Maurice}

Service de Chirurgie Générale et Digestive, CHUYO, Burkina Faso

\section{Traoré Alain Ibrahim}

Service d'anesthésie réanimation CHUSS Bobo-Dioulasso, Burkina Faso

Doi: 10.19044/esj.2017.v13n36p424 URL:http://dx.doi.org/10.19044/esj.2017.v13n36p424

\begin{abstract}
Title: Surgical management of abdominal incisional hernia with mesh placement: short- term experience of the University Hospital Yalgado Ouedraogo Introduction: The incisional hernia repair has several variants depending on the implantation site of the mesh. It may be intraperitoneal, preperitoneal, pre-mucosal or pre-musculoskeletal. Objective: The aim of the work was to report at short term the experience of the general and digestive surgery service of the Yalgado Ouédraogo University Hospital Center for the surgical management of abdominal incisional hernia with mesh placement in retro-muscular prefascial Patients and methods: This was a cross-sectional and descriptive study with a review of the files and a survey of 17 patients operated on for voluminous abdominal incisional hernia (diameter greater than $10 \mathrm{~cm}$ ) from September 1, 2014 to August 31, 2015. Results: The mean duration of hospitalization was 7 days with extremes of 4 and 15 days. The dressings lasted on average 7 days with extremes of 4 and 15 days. The suction drains remained in place on average 8.56 days with extremes of 5 and 14 days and the mean duration of the abdominal wall contusion was 24.5 days with extremes of 10 and 40 days.
\end{abstract}


The early postoperative complications were minor: 8 seromas, 1 hematomas, 1 superficial infection of the wall. Patients were followed for an average duration of 9.47 months with extremes of 4 and 14 months. The complications during this period were: 3 minor residual pain (limited to the scar) and 1 recurrence. Thirteen patients (76.5\%) were satisfied with the intervention. Mortality was zero. Conclusion: Short time complications related to the surgical management of abdominal incisional hernia with mesh placement in retro-muscular prefascial in CHUYO are mainly minor.

Keywords: Retromuscular mesh repair; incisional hernia repair, complication, recurrence

\section{Résumé}

Titre: Traitement chirurgical des éventrations abdominales avec pose de prothese: experience à court terme du centre hospitalier universitaire Yalgado Ouédraogo Introduction: La cure d'eventration a plusieurs variantes selon le site d'implantation des prothèses. Il peut être intrapéritonéal, prépéritonéal, rétromusculaire préfascial ou prémusculopréfascial. Objectif : Le but de l'étude était de rapporter l'expérience à court terme du service de chirurgie générale et digestive du Centre Hospitalier Universitaire Yalgado Ouédraogo dans le traitement chirurgical des larges éventrations avec pose de prothèse en retro-musculaire préfascial. Patients et méthodes : Il s'est agi d'une étude transversale et descriptive avec revue des dossiers et enquête auprès de 17 patients opérés pour volumineuses éventrations (diamètre supérieur à $10 \mathrm{~cm}$ ) du $1^{\mathrm{er}}$ septembre 2014 au 31 Août 2015. Résultats: La durée moyenne d'hospitalisation était de 7 jours avec des extrêmes de 4 et 15 jours. Les pansements ont duré en moyenne 7 jours avec des extrêmes de 4 et 15 jours. Les drains aspiratifs étaient restés en place en moyenne 8, 6 jours avec des extrêmes de 5 et 14 jours et la durée moyenne de la contension de la paroi abdominale était de 24,5 jours avec des extrêmes de 10 et 40 jours. Dix cas de complications postopératoires précoces ont été notées, à savoir, 8 séromes, 1 hématome, 1 infection superficielle de la paroi. Les patients ont été suivis sur une durée moyenne de 9,5 mois avec des extrêmes de 4 et 14 mois. Les complications, pendant cette période étaient représentées par 3 cas de douleurs abdominales résiduelles mineures et 1 récidive. Treize patients (76,5\%) se disaient satisfaits de l'intervention. La mortalité était nulle. Conclusion : les complications à court terme liées à la pose de prothèse retromusculaire préfasciale au CHUYO sont majoritairement mineures.

Mots-clés : Prothèse rétro-musculaire, éventration, complication, récidive 


\section{Introduction}

Les éventrations représentent l'une des complications classiques de la chirurgie abdominale (Bouillot et al 2012). L'usage des prothèses dans leur prise en charge est devenu aujourd'hui le Gold standard (Poussier $\mathrm{M}$ et al 2013; Kokotovic et al 2016). En Afrique, et particulièrement au Burkina Faso, comme au Sénégal, la pariétoplastie prothétique tend progressivement à être le traitement de référence (Diao et al 2012). Cette technique de cure a plusieurs variantes selon le site d'implantation des prothèses qui peut être intrapéritonéal, prépéritonéal, rétromusculaire préfascial ou prémusculopréfascial (Bouillot 2004). La variante la plus utilisée dans notre contexte de travail est la prothèse en retro-musculaire préfascial. Le but de ce travail était de rapporter notre expérience, au service de chirurgie générale et digestive du CHUYO.

\section{Patients et Méthodes}

Il s'est agi d'une étude transversale, descriptive avec revue des dossiers et enquête auprès de tous les patients opérés pour volumineuses éventrations abdominales (grand axe du collet supérieur à $10 \mathrm{~cm}$ ), avec pose de prothèse en position retro-musculaire pré-fascial et suivis régulièrement de sepembre 2014 à août 2015 dans le service de chirurgie générale et digestive du Centre Hospitalie Universitaire Yalgado Ouédraogo (CHUYO). Trois patients porteurs d'une large éventration, ayant bénéficié de la cure par prothèse en retro-musculaire pré-fascial, ont été exclus car perdus de vue pendant la période de l'étude. Au total, 17 patients ont été inclus dans l'étude. Il s'agisait de 11 femmes et 6 hommes dont l'âge moyen était de 36,6 ans avec des extrêmes de 16 et 60 ans. Six de ces patients présentaient une co-morbidité dont quatre étaient une obésité. Des soins locaux de la paroi abdominale antérieure au savon simple ou à la Bétadine ${ }^{\circledR}$ étaient systématiquement réalisés en période préopératoire, la veille et le jour de l'intervention. L'antibioprophylaxie était systématique à base de Ceftriaxone ${ }^{\circledR}$. Par contre le régime de perte pondérale n'était pas institué chez nos malades obèses. Tous nos patients ont bénéficié d'une laparotomie sous anesthésie générale. Aucun patient n'a été opéré en urgence. L'incision cutanée était médiane, fusiforme emportant l'ancienne cicatrice. Après la dissection et la résection du sac, une adhésiolyse intrapéritonéale était réalisée en cas d'adhérences. La prothèse de polypropylène était mise en place en position retro-musculaire préfasciale après avoir décollé le fascia musculaire postérieur des muscles larges de l'abdomen. La prothèse était fixée à l'aide de points séparés, transfixiant l'épaisseur musculoaponévrotique en $U$, et noués dans le plan de décollement sous cutané prémusculoaponévrotique au fil non résorbable $n^{\circ} 2.0$. Deux drains aspiratifs de Redon, étaient placés respectivement dans l'espace retro-musculaire au 
contact de la prothèse (figure 1) et dans l'espace de décollement préaponévrotique sous-cutanée. Une contension de la paroi abdominale antérieure à l'aide d'une bande élastique adhésive a été réalisée systématiquement. Le dégré de satisfaction des malades sur l'amelioration de leur qualité de vie post-opératoire était apprécié. Les paramètres étudiés étaient les incidents per-opératoires, les complications post-opératoires précoces, secondaires et tardives, les soins post-opératoires (la durée des pansements, la durée d'hospitalisation, la durée du drainage et de la contension de la paroi abdominale), le suivi (la durée du suivi et le degré de satisfaction de la qualité de vie en post-opératoire) et le décès. L'étude a été autorisée par la comité national d'éthique pour la recherche en santé du Burkina Faso. Une autorisation a été obtenue auprès de chaque patient pour son inclusion dans l'étude. La collecte de données s'est faite de façon anonyme et la confidentialité a été respectée pour tous les patients.

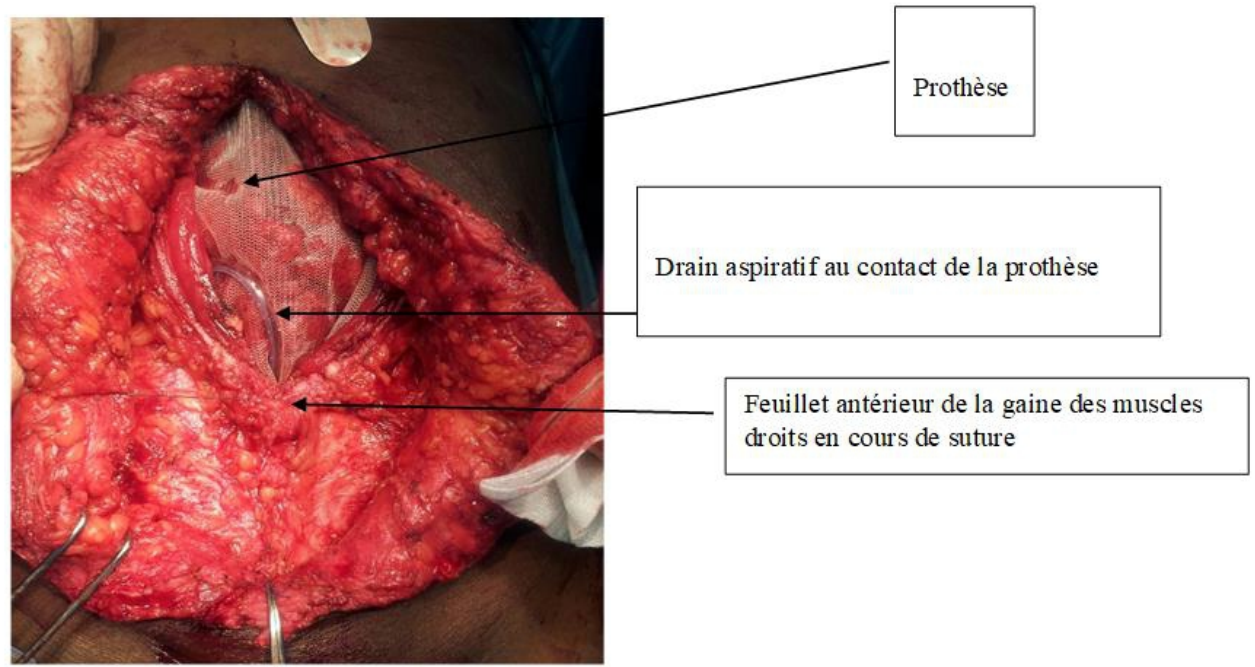

\section{Resultats}

Les soins post-opératoires: cinq patients ont bénéficié de la prévention thrombo-embolitique à base d'Enoxaparine ${ }^{\circledR}$. La durée moyenne de l'hospitalisation était de 7 jours avec des extremes de 4 et 15 jours. Le durée moyene du drainage était de 8,6 jours avec des extrêmes de 5 et 14 jours et celle de la contension de la paroi abdominale était de 24,5 jours avec des extrêmes de 10 et 40 jours.

Les incidents per-opératoires étaient représentés par une plaie pariétale iléale accidentelle sans ouverture de la muqueuse qui a occasionné une suture immédiate. 


\section{Les complications}

Treize patients dont dix femmes et trois hommes, ont présenté des suites opératoires compliquées. Les complications étaient précoces dans 10 cas (huit cas de sérome, un cas d'hématome et un cas d'infection pariétale superficielle). Une patiente avait présenté à la fois un sérome, et une infection sous-cutanée. Ces complications ont été traitées par des soins locaux avec une suture cutanée secondaire dans un cas. Les complications étaient tardives dans 04 cas : 01 récidive et 03 cas de douleurs abdominales résiduelles mineures cotées sur l'échelle visuelle analogique score à $2 / 10$. La récidive était survenue chez une patiente qui avait une co-morbidité à type d'obésité morbide avec un IMC de 39,06.

Le suivi post-opératoire: les patients ont été suivis sur une durée moyenne de 9,5 mois avec des extrêmes de 4 et 14 mois. Au terme du suivi, 13 patients étaient satisfaits de la qualité de leur vie post-opératoire à l'interrogatoire. Par contre 4 patientes se disaient non satisfaites du fait de la survenue de complications tardives. La mortalité était nulle.

\section{Discussion}

La technique de cure d'éventration par pose de prothèse en retromusculaire préfaciale, comporte peu d'infection atteignant la prothèse et peu de récidives (Bouillot JL et al 2004; Lechaux JP et al 2004). Ces avantages, ont justifié notre choix comme technique de référence dans la cure d'éventration dans notre milieu. Bien que l'abord laparoscopique en général s'impose progressivement dans notre pays depuis 2005 (Bonkoungou et al 2009), cette voie d'abord proposée par certains auteurs dans le traitement de l'éventration (Grant et al 2002; Heniford et al 2003) n'est pas encore utilisée dans notre pays pour cette indication. Nous avons noté en per-opératoire une plaie accidentelle du grêle sans ouverture muqueuse, ce qui a autorisé la pose de prothèse. Des lésions viscérales peropératoires ont été également rapportée par certains auteurs. En effet, Bisgaard $\mathrm{T}$ et al (Bisgaard et al 2009) au Danemark, trouvait que $0,9 \%$ de complications viscérales. L'utilisation de la prothèse à base de polypropylène chez tous nos patients tient à la disponibilité et à l'accessibilité facile de ce type de prothèse dans notre milieu d'excercice. Dans le soucis de prevenir les complications infectieuses post-opératoires, nous avons pratiqué l'antibioprophylaxie. Ailleurs , Harouna et Mathonnet (Harouna et al 2001; Mathonnet et al 1998), l'ont pratiquée. Cependant cette pratique est encore discutée (Gillion et Palot 2015).

Les complications post-opératoires précoces survenus chez nos patients étaient mineures, représentées par 8 séromes, 1 hématome, 1 infection pariétale superficielle. En plus de ces complications, d'autres complications comme la fistule entéro-cutanée, l'absence de cicatrisation 
cutanée et l'abcès superficiel ont été rapportées par S Rohr (Rohr et al 2010). Nous n'avons pas noté d'infection du matériel prothétique, qui est une complication redoutée des cures d'éventration avec pose de prothèse. Elle a été rapportée par Boullenois (Boullenois 2016).

Nos patients ont été hospitalisés pendant sept jours en moyenne en postopératoire. Horhant et col (Horhant et al 1996) en France et Ott V (Ott et al 2003) en Suisse trouvaient une durée moyenne respective de 10 et 15 jours. Notre durée moyenne d'hospitalisation relativement courte pourrait s'expliquer par l'absence de complications majeures chez nos patients pouvant nécessiter des soins plus longs. Tous nos patients avaient bénéficié d'un drainage type aspiratif, pendant une durée moyenne de 8,6 jours. Harouna YD et col au Niger (Harouna et al 2001) observaient un drainage aspiratif de trois jours. La différence s'explique par le fait que dans notre série la technique chirurgicale a nécessité un décollement à deux niveaux provoquant une production importante de sérosité en post opératoire. La contention abdominale par bandage à l'élastoplaste a été systematique chez tous nos patients avec une durée moyenne de 24,5 jours. Nordback P et Vuilleumier H (Nordback P et Vuilleumier 2006) en Suisse préconisaient une compression par bandage abdominale pour une durée moyenne de deux semaines (entre une à quatre semaines). La durée a été prolongée chez nous parce qu'il s'agissait d'une technique avec des décollements importants pourvoyeurs de complications à type de formation de séromes ou d'hématomes. Nos patients ont été suivi sur une durée moyenne de 9,47 mois. Les complications survenues à cette periode, étaient essentiellement des douleurs abdominales résiduelles mineures (03 cas). Ces douleurs se justifient non seulement par le mode de fixation de la prothèse dans la technique, qui engendre une séquestration de filets nerveux perforants de la paroi, responsable de la douleur ; mais aussi par le type de prothèse utilisé dans notre série, la prothèse macroporeuse à base de polypropylène, qui est considérée comme étant rigide et source de douleur post opératoire par rapport à la prothèse à polyester (Bouillot et al 2004). Nous avons noté dans notre série un cas de récidive (5\%) avec un recul moyen court de 9,47 mois. Ailleurs Poghosyan T et al (Poghosyan at al 2012) en France ont rapporté un taux de récidive de 3\% sur un recul moyen de 5 ans. La mortalité était nulle dans notre série comme dans les séries de Berrada et al (Berrada et al 1996) au Maroc et Cubertafond et al (Cubertafond et al 1998) en France. Par contre , Gillion JF et Palot JP (Gillion et Palot 2015) ont rapporté un cas de décès après une infection profonde.

\section{Conclusion}

Les complications liées à la pose de prothèse retromusculaire préfasciale au CHUYO sont des complications mineures. La réalisation 
d'une étude prospective avec un effectif plus important et un recul plus long permettrait de mieux évaluer les complications tardives.

\section{References:}

1. Bouillot JL, Pogoshian T, Corigliano N, Canard G, Veyrie N. Traitement des volumineuses éventrations abdominales. Jchirv 2012;149 (5): 55-61

2. Poussier M, Denève E, Blanc $P$, Boulay E, Bertrand M, Nedelcu M, Herrero A, Fabre JM, Nocca D. Les différents matériaux prothétiques pour la réparation pariétale abdominale. Jchirv 2013 150, (1)55-62

3. Kokotovic D, Bisgaard T, Helgstrand F. Prospective nationwide study on long term mesh related complications after incisional hernia repair.J.jamcollisurg 2016; 06: 355

4. Diao ML, Tendeng JN, Manyacka Ma Nyemb P, Ehode C, Wade TMM, Ndaw AL, Cissé M, Konaté I, Touré CT. La cure par prothèse des éventrations abdominales : à propos de 15 cas. Revue africaine de chirurgie et spécialités $2012 ; 5$ (3) :1-8

5. Bouillot J.L. Traitement des éventrations abdominales par prothèse de Dacron ${ }^{\circledR}$ rétromusculaire. Jchirv 2004 ; 141(4): 233-7

6. Bouillot JL, Servajean S, Berger N, Veyrie N, Hugol D. Comment choisir une prothèse pour le traitement des éventrations abdominales ? Annales de chirurgie 129 (2004) 132-7.

7. Lechaux JP, Lechaux D, Chevrel JP. Traitement des éventrations de la paroi abdominale. EMC chirurgie 2004;1 : 601-9.

8. Bonkoungou G, Sanou A, Sam T, Bonkoungou Z, Zida M, Traoré S. La cholecystectomie laparoscopique au Burkina Faso: à propos de 32 cas. Journal de coelichirurgie 2009; 71: 57-9

9. Grant AM, EU Hernia Trialists Collaboration. Laparoscopic versus open groin hernia repair: meta-analysis of randomised trials based on individual patient data. Hernia. 2002;6:2-10.

10. Heniford B.T, Park A, Ramshaw B.J, and Voeller G. Laparoscopic repair of ventral hernias: nine years' experience with 850 consecutive hernias. Annals of surgery 2003; 238(3):391.

11. Bisgaard T, Kehlet H, Bay-Nielsen MB, et al. Nationwide study of early outcomes after incisional hernia repair. Br J Surg 2009; 96:1452-7.

12. Harouna YD, Rakotomalala J. La volumineuse éventration médiane en zone tropicale : étiologies et résultats du traitement par la technique de Judd. Annales de chirurgie plastique 2001 ; 46 : 595-8.

13. Mathonnet M, Antarieu S, Gainant A, Preux PM, Boutros-Toni F, Cubertafond P. Eventrations post opératoires : prothèses intra ou extra-péritonéales. Chirurgie 1998 ; 123 (2) : 154-61. 
14. Gillion JF, Palot JP. Éventrations - prothèses infectées : traitement et prévention Abdominal Wall Incisional Hernias - Infected Prosthesis: Treatment and Prophylaxis. e-mémoires de l'Académie Nationale de Chirurgie 2015, 14 (2) : 069-080

15. Rohr. S, Arnaud JP, Mathonnet M, Bouvier S, Desrousseaux B, Baulieux J. Le traitement d'éventrations abdominales employant une prothèse Parietex ${ }^{\circledR}$ composite (164). Jchirv 2010 ; 147, (4) $1: 14-5$

16. Boullenois H, Moszkowicz D, Poghosyan T, Bouillot JL. Surgical management of chronic mesh infection following incisional hernia repair. Journal of Visceral Surgery 2016. 153, 461-4

17. Horhant P, Ledu J, Chaperon J, Lavenac G, Mambrini A. Traitement des éventrations post opératoires par prothèses non résorbables. Journal de Chirurgie $1996 ; 7$ : 311-6.

18. Ott V. Cure d'éventrations avec renforcement par un filet de Mersilène en position prépéritonéale. Université de Génève. Thèse de Médecine $2003: 55 \mathrm{p}$.

19. Nordback P, Vuilleumier H. Cures d'éventration et des hernies ombilicales volumineuses par cœlioscopie : techniques et résultats. Rev Med Suisse 2006 ; (2) : 314-36

20. . Poghosyan T, Veyrie N, Corigliano N, Helmy N, Servajean S, Bouillot JL. Retromuscular mesh repair of midline incisional hernia with polyester standard mesh: monocentric Experience of 261 consecutive patients with a 5-year follow-up. World. J Surg $2012 ; 36$ : 782-90.

21. Berrada S, EL Mouatacim K, Kadiri B. Réparation prothétique des éventrations post-opératoires. Médecine du Maghreb 1996 ; 57 : 324.

22. Cubertafond P, Sava P, Gainant A, Ugazzi A. Cure chirurgicale des éventrations post-opératoires par plaques prothétiques. Journal de Chirurgie $1998 ; 115$ : 66-71. 